\title{
Increased in vivo angiogenic effect of glioma stromal mesenchymal stem-like cells on glioma cancer stem cells from patients with glioblastoma
}

\author{
BYUNG HO KONG $^{1 *}$, HYUN-DO SHIN ${ }^{3 *}$, SE-HOON KIM ${ }^{4}$, HYUN-SU MOK ${ }^{2}$, JIN-KYOUNG SHIM ${ }^{5}$, \\ JI-HYUN LEE ${ }^{5}$, HYE-JIN SHIN ${ }^{2}$, YONG-MIN HUH ${ }^{6}$, EUI-HYUN KIM ${ }^{5}$, EUN-KYUNG PARK ${ }^{5}$, \\ JONG HEE CHANG ${ }^{5}$, DONG-SEOK KIM ${ }^{5}$, YONG-KIL HONG ${ }^{2}$, \\ SUN HO KIM ${ }^{5}$, SU-JAE LEE ${ }^{7 * *}$ and SEOK-GU KANG ${ }^{\text {*** }}$ \\ Departments of ${ }^{1}$ Medical Science and ${ }^{2}$ Neurosurgery, The Catholic University of Korea College \\ of Medicine, Seoul; ${ }^{3}$ Department of Medical Science, Seonam University College of Medicine, Namwon; \\ Departments of ${ }^{4}$ Pathology, ${ }^{5}$ Neurosurgery and ${ }^{6}$ Radiology, Severance Hospital, Yonsei University College \\ of Medicine, Seoul; ${ }^{7}$ Deparment of Chemistry, Hanyang University, Seoul, Republic of Korea
}

Received December 20, 2012; Accepted February 21, 2013

DOI: 10.3892/ijo.2013.1856

\begin{abstract}
The presence of glioma stromal mesenchymal stem-like cells (GS-MSLCs) in tumors from glioma patients has been previously reported. The mechanisms through which these cells function as a part of the glioma microenvironment, however, remain incompletely understood. We investigated the biological effects of GS-MSLCs on glioma cancer stem cells (gCSCs), testing the hypothesis that GS-MSLCs alter the biological characteristics of gCSCs. GS-MSLCs and gCSCs were isolated from different glioblastoma (GBM) specimens obtained from patients. In in vitro experiments, gCSCs were cultured alone or co-cultured with GS-MSLCs, and gCSCs cell counts were compared between the two groups. In addition, two groups of orthotopic GBM xenografts in mice were created, one using gCSCs from the monoculture group and one using gCSCs isolated from the co-culture group, and tumor volume and survival were analyzed. Furthermore, in vivo proliferation, apoptosis and vessel formation were examined using immunohistochemical analyses. In vitro cell counts for gCSCs co-cultured
\end{abstract}

Correspondence to: Professor Su-Jae Lee, Department of Chemistry, Hanyang University, 17 Haendang-dong, Seongdong-gu, Seoul 133-791, Republic of Korea

E-mail: sj0420@hanyang.ac.kr

Dr Seok-Gu Kang, Department of Neurosurgery, Severance Hospital, Yonsei University College of Medicine, 50 Yonsei-ro, Seodaemun-gu, Seoul 120-752, Republic of Korea

E-mail: seokgu9@kornet.net

*Contributed equally as first authors

${ }^{* *}$ Contributed equally as senior authors

Key words: angiogenesis, glioma, glioma cancer stem cells, glioma stroma, mesenchymal stem like cells, microenvironment with GS-MSLCs increased 3-fold compared to gCSCs cultured alone. In orthotopic xenograft experiments, mice injected with gCSCs isolated from the co-culture group had significantly larger tumor volume, measured on day 40 after injection, and their survival times were shorter. Immunohistochemical analysis showed increased tumor expression of CD31, indicative of enhanced microvessel formation in mice injected with gCSCs co-cultured with GS-MSLCs compared to mice injected with gCSCs cultured alone. However, proliferation (PCNA) and apoptosis (TUNEL) markers showed no significant difference between the two groups. In conclusion, GS-MSLCs may influence the biological properties of gCSCs, shifting them towards a more aggressive status; moreover, increased angiogenesis may be a critical component of this mechanism.

\section{Introduction}

The influence of the cancer microenvironment on the behavior of tumors has received considerable attention since Poste and Fidler $(1,2)$ re-illuminated 'seed and soil' hypothesis, first postulated by the British surgeon Paget (3). The cancer microenvironment, which consists of variety of cell types, the extracellular matrix and signaling molecules, significantly affects the initiation, progression and metastasis of cancers $(4,5)$. Among these components of the tumor microenvironment, mesenchymal stem cells (MSCs) or mesenchymal stem-like cells (MSLCs) have been focus of particular research interest.

With accumulating evidence suggesting a strong association between MSCs and various tumors, including breast cancer $(6,7)$, lipoma (8), gastric cancer (9) and bone sarcomas (10), the veiled relationship between MSCs and tumors has started to come into focus. Tumors of the human brain are no exception. Lang et al first described that cells which adhere and grow on plastic; differentiate into osteocytes, adipocytes and chondrocytes; and express the appropriate MSC surface markers isolated from surgical glioma specimens [Society for Neuro-Oncology 
Annual Meeting, Neuro Oncol 9: abs. 596, 2007, and ASCO Annual Meeting, J Clin Oncol 26 (Suppl 15): abs. 2001, 2008].

Moreover, a series of studies reported the isolation of cells resembling MSCs from normal mouse brain (11), glioma xenograft specimens (12) and Korean glioma specimens (13). These cells, referred to as MSLCs, do not completely meet all the MSC-defining criteria proposed by the International Society for Cellular Therapy (14). However, notwithstanding their glioma origin, they have properties similar to those of MSCs, such as adherence to plastic, expression of surface antigens characteristic of MSCs, mesenchymal differentiation potential and a lack of gliomagenesis potential.

Given that MSCs in the tumor microenvironment play a critical role in determining the biological behavior of the tumor (15), the fact that MSLCs are present in glioma specimens and share characteristics of MSCs suggests that these cells also likely make an important contribution to the tumor microenvironment. Hossain et al recently presented that tumor-associated mesenchymal stromal cells in glioblastomas (GBMs) enhance the tumorigenic and proliferative properties of glioma cancer stem cells ( $\mathrm{gCSCs}$ ) through the interleukin (IL)-6/STAT3 (signal transducer and activator of transcription 3) pathway [Society for Neuro-Oncology annual meeting, Neuro Oncol 13 (Suppl 3): abs. iii19, 2011]. Moreover, changes in the microenvironment have been clearly shown to affect the biological characteristics of gCSCs (16). The exact functions of MSCs and MSLCs, however, are a matter of controversy.

In this study, we investigated the possible role of MSLCs in GBM by examining how glioma stroma mesenchymal stem-like cells (GS-MSLCs) obtained from Korean glioma specimens affect gCSCs, which are thought to be the major cells responsible for the initiation, maintenance, and progression of glioma (17-22). We hypothesized that GS-MSLCs favor tumor growth by altering the biological nature of gCSCs. To verify this, we transfected gCSCs with green fluorescent protein (GFP) and cultured them alone or together with GS-MSLCs. After 3 weeks in culture, we compared the numbers of GFP-emitting gCSCs (GFP-gCSCs) obtained from the two culture groups. Finally, we prepared orthotopic xenograft mouse models using GFP-gCSCs cultured alone and GFP-gCSCs isolated from GS-MSLCs co-cultures, and then compared survival, tumor volume, tumor cell proliferation and apoptosis, and microvessel density between the two groups.

\section{Materials and methods}

Isolation and culture of human gCSCs and GS-MSLCs. Specimens for isolation of gCSCs were collected in the operating room from GBM patients undergoing surgery. Approval for harvest and use was obtained from the institutional review boards of Severance Hospital, Yonsei University College of Medicine (4-2012-0212), and Seoul St. Mary's Hospital, the Catholic University of Korea College of Medicine (KC10SNS10466). Informed consent was provided according to the Declaration of Helsinki. Neuropathologists diagnosed each surgical specimen according to world health organization (WHO) classifications (23). gCSCs were isolated from GBM specimens within one hour of glioma removal using a previously described mechanical dissociation method for isolating gCSCs from human brain $(19,22,24)$. Briefly, surgical speci- mens were minced and dissociated with a scalpel in Dulbecco's modified Eagle's medium/Nutrient Mixture F-12 (DMEM/F-12; Mediatech, Manassas, VA, USA) and then passed through a series of $100-\mu \mathrm{m}$ nylon mesh cell strainers (BD Falcon, Franklin Lakes, NJ, USA). Cell suspensions were washed twice in DMEM/F-12 and cultured in gCSC complete medium (DMEM/F-12) containing 1X B27 (Invitrogen, San Diego, CA, USA) plus $20 \mathrm{ng} / \mathrm{ml}$ basic fibroblast growth factor (bFGF; Sigma, St. Louis, MO, USA), $20 \mathrm{ng} / \mathrm{ml}$ epidermal growth factor (EGF; Sigma), and $50 \mathrm{U} / \mathrm{ml}$ penicillin $/ 50 \mathrm{mg} / \mathrm{ml}$ streptomycin (Gibco, Invitrogen Korea, Seoul, Korea). One type of gCSC (gCSC0504) from GBM was used for this study (22).

Methods shown to be reliable for isolating MSLCs from normal brain (11), orthotopic glioma xenografts (12) and Korean glioma specimens (13) were used for isolation of GS-MSLCs from GBM specimens. Cells were isolated within one hour of glioma removal using the mechanical dissociation method described above for gCSCs. Cell suspensions were washed twice in minimal essential medium- $\alpha$ (MEM $\alpha$; Mediatech, Herndon, VA, USA), placed in a $10-\mathrm{cm}^{2}$ cell culture dish at a density of $2 \times 10^{6}$ cells $/ \mathrm{cm}^{2}$, and cultured in complete MSC medium consisting of MEM $\alpha$ (Mediatech), $10 \%$ fetal bovine serum (FBS; Lonza, Basel, Switzerland), $2 \mathrm{mM}$ L-glutamine (Mediatech) and antibiotic-antimycotic solution (Gibco, Invitrogen Korea, Seoul, Korea). After 24 h, non-adherent cells were removed by washing twice with phosphate-buffered saline (PBS; Mediatech) and the adherent cells were cultured until they reached confluence. Cells were then trypsinized $(0.25 \%$ trypsin with $0.1 \%$ EDTA) and subcultured at a density of 5,000 cells $/ \mathrm{cm}^{2}$. Cells were cultured continuously through three passages, consistent with their status as progenitor/stem cells. Cell morphology was examined by observing cell cultured with inverted phase-contrast microscope (IX71 Inverted Microscope; Olympus, Tokyo, Japan). One type of GS-MSLCs (KGS-MSC0503) form GBM was used for this study (13).

Lentiviral vector transduction and expression. GFP-gCSCs for cell counting were generated by growing gCSCs in complete medium and then applying GFP-expressing lentiviral supernatants. Polybrene (Sigma) was added to a final concentration of $8 \mu \mathrm{g} / \mathrm{ml}$ and incubated with cells for $18 \mathrm{~h}$. After infection, the cells were placed in fresh growth medium and cultured in a standad manner. Cells were treated with $1 \mathrm{mg} / \mathrm{ml}$ puromycin (Life Technologies Korea, Seoul, Korea) to eliminate uninfected cells and generated stable GFP-gCSC. GFP-expressing $\mathrm{gCSCs}$ were isolated for use in further experiments.

Sorting and counting of GFP-gCSCs. Increase in cell numbers was evaluated in GFP-gCSCs cultured alone or together with GS-MSLCs for 3 weeks. For the GFP-gCSCs monoculture group, complete MSC medium (without GS-MSLCs) was incubated for $24 \mathrm{~h}$ (Fig. 1A), after which the medium was removed, plates were washed three times with PBS, and fresh gCSCs complete medium containing $1.25 \times 10^{5}$ GFP-gCSCs was added (Fig. 1B). The medium was replaced with fresh gCSCs complete medium every 3 days. After 3 weeks, gliomasphere formation by GFP-gCSCs was evident (Fig. 1C). For the GFP-gCSCs/GS-MSLC co-culture group, GS-MSLCs $\left(2.5 \times 10^{5}\right)$ were first isolated and seeded in $100-\mathrm{mm}$ culture 

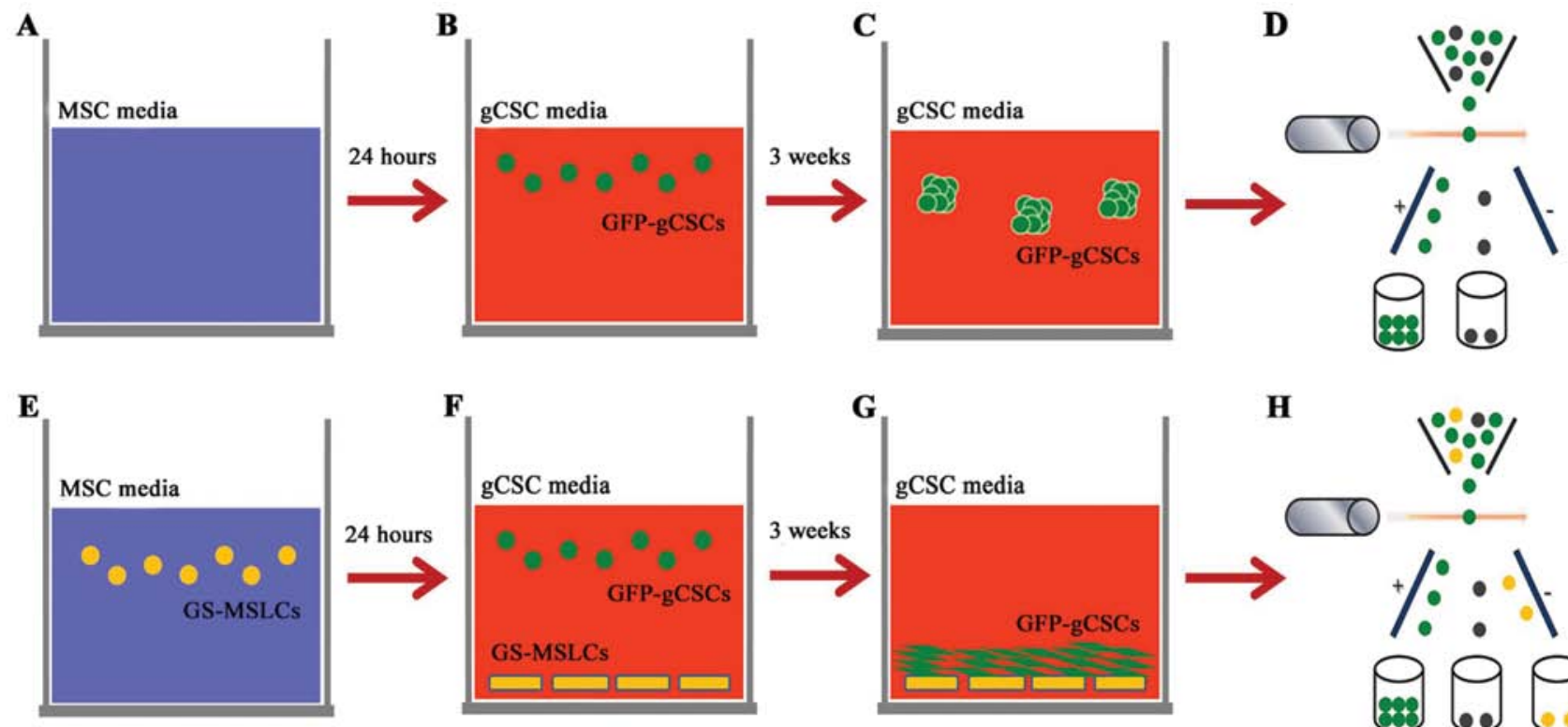

$\mathbf{H}$

Figure 1. Cell culture, sorting and counting of GFP-gCSC monoculture and GFP-gCSC/GS-MSLC co-culture groups. (A-D) For the monoculture group, (A) culture dishes were filled with complete MSC medium (without GS-MSLCs). (B) After $24 \mathrm{~h}$, the MSC medium was replaced with complete gCSC medium containing GFP-gCSCs. (C) After 3 weeks of culture, formation of GFP-gCSC gliomaspheres was evident. (D) GFP-gCSCs from the monoculture group were counted and sorted by FACS analysis. (E-H) For the GFP-gCSC/GS-MSLC co-culture group, (E) GS-MSLCs were seeded in complete MSC medium. (F) After $24 \mathrm{~h}$, the MSC medium was replaced with gCSC complete medium containing GFP-gCSCs. (G) After 3 weeks of culture, GFP-gCSCs had adhered to GS-MSLCs on the bottom of the culture dish. (H) GFP-gCSCs from the GS-MSLC co-culture group were counted and sorted by FACS analysis.

dishes containing MSC complete medium (Fig. 1E). The next day, the culture medium was removed and cells were washed three times with PBS. gCSC complete medium containing $1.25 \times 10^{5}$ GFP-gCSCs were then added (Fig. 1F), and cells were incubated at $37^{\circ} \mathrm{C}$ in a humidified $5 \% \mathrm{CO}_{2}$ incubator for 3 weeks. The medium was replaced with fresh gCSC complete medium every 3 days. After 3 weeks, GFP-gCSCs had adhered to GS-MSLCs at the bottom of the culture dish (Fig. 1G). In the last step, GFP-gCSC monoculture (Fig. 1D) and GS-MSC co-culture (Fig. 1H) groups were isolated by fluorescence-activated cell sorting (FACS).

Orthotopic glioma xenograft model. Male athymic nude mice (4-8-weeks-old; Central Laboratory Animal Inc., Seoul, Korea) were used for tumor xenograft experiments. Mice were housed in micro-isolator cages under sterile conditions and observed for at least 1 week before study initiation to ensure proper health. Temperature, lighting and humidity were controlled centrally. All experimental procedures were approved by Yonsei University College of Medicine Institutional Animal Care and Use Committee and the Catholic University of Korea College of Medicine Institutional Animal Care and Use Committee. Mice were anesthetized with a solution of Zoletil (30 mg/kg; Virbac Korea, Seoul, Korea) and xylazine (10 mg/kg; Bayer Korea, Seoul, Korea) delivered intraperitoneally. GFP-gCSCs $\left(5 \times 10^{5}\right)$ isolated from monoculture or $\mathrm{gCSC} / \mathrm{GS}-\mathrm{MSLC}$ co-culture groups were implanted into the right frontal lobe via a Hamilton syringe (Dongwoo Science Co., Seoul, Korea) inserted to a depth of $4.5 \mathrm{~mm}$ using a guide-screw system, as described previously (25). Cells from each culture condition were simultaneously injected into 10 mice at a rate of $0.5 \mu \mathrm{l} / \mathrm{min}$ using a multiple micro-infusion syringe pump (Harvard Apparatus, Holliston, MA, USA). The body weights of mice were checked every other day. If body weight decreased by more than $15 \%$ compared to the original body weight, mice were euthanized according to the study protocol.

Tumor volume measurement. For comparison of the tumor volume, eight GFP-gCSC-injected mice (four each from monoculture and GS-MSLC co-culture groups) were sacrificed 40 days after injection. The brains were carefully removed and placed in $10 \%$ buffered formalin for fixation. The fixed brains were then cut axially every $2 \mathrm{~mm}$ and embedded in paraffin. Tumor volume was calculated by measuring the section with the largest tumor portion and applying the formula, length $\mathrm{x}$ width $^{2}$ x $0.5(26,27)$.

Immunohistochemical analysis of PCNA, TUNEL and $C D 31$. Paraffin-embedded tissues were immunostained for proliferating cell nuclear antigen (PCNA) and the endothelial cell-specific marker CD31 to evaluate tumor cell proliferation and microvessel density, respectively. Apoptosis was assessed using deoxynucleotidyl transferase-mediated dUTP nick-end labeling (TUNEL) assays. Tissue sections (4-6 $\mu \mathrm{m}$ thick) were mounted on silanized glass slides and dried overnight. The sections were deparaffinized using xylene, then treated with graded series of alcohol $\left[100,95\right.$ and $80 \%$ ethanol $/ \mathrm{ddH}_{2} \mathrm{O}$ ( $\mathrm{vol} / \mathrm{vol})]$ and rehydrated in PBS (pH 7.5). For PCNA immunostaining, sections were microwaved for $5 \mathrm{~min}$ in water followed by treatment with $3 \% \mathrm{H}_{2} \mathrm{O}_{2}$ in methanol for $5 \mathrm{~min}$ to block endogenous peroxidase. Slide-mounted slices were then incubated at $4^{\circ} \mathrm{C}$ overnight with mouse, anti-PCNA primary antibody (1:100; Invitrogen Korea, Seoul, Korea) in 


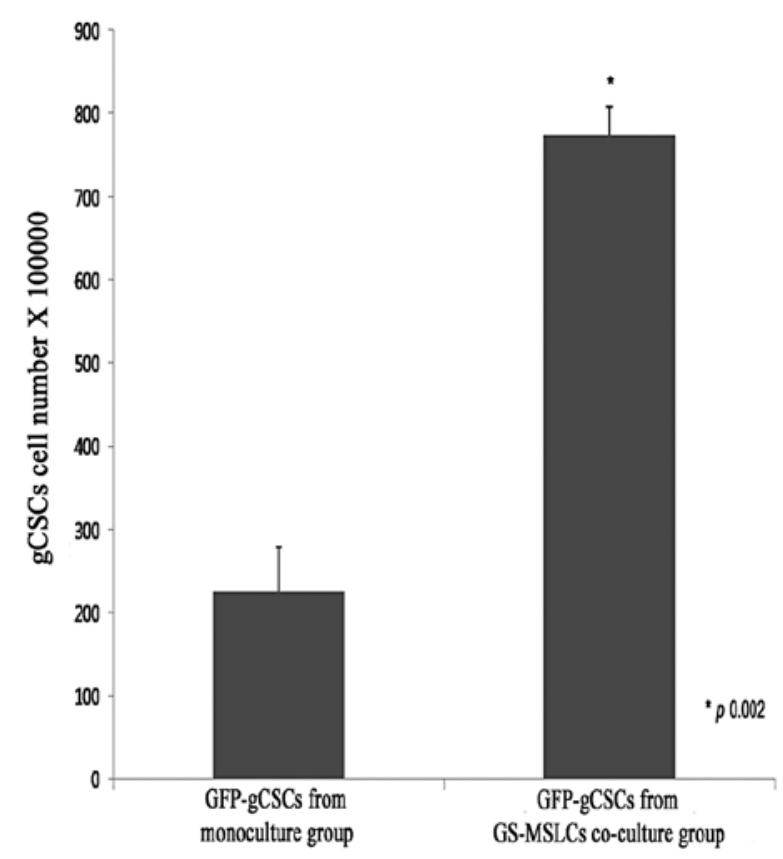

Figure 2. Comparison of GFP-gCSC cell counts. The numbers of GFP-gCSCs isolated from monoculture and GS-MSLC co-culture groups were compared. GFP-gCSC cell counts were significantly increased in the GS-MSLC co-culture group $\left(7.76 \pm 0.39 \times 10^{5}\right)$ compared to the GFP-gCSC monoculture group $\left(2.62 \pm 0.44 \times 10^{5} ; \mathrm{p}=0.002\right)$.

a $5 \%$ normal horse serum, $1 \%$ normal goat serum phosphate buffer solution. Peroxidase-conjugated secondary antibodies were used and visualized by incubating the slides with 3, 3'-diaminobenzidine (DAB) stable substrate solution for 10-20 min. The sections were rinsed with distilled water, counterstained with Gill's hematoxylin for $1 \mathrm{~min}$, and mounted with Universal Mount (Research Genetics, Huntsville, AL, USA). Twenty different areas of a single slide (one brain section) were scored. Expression of CD31 (1:50; BD Pharmigen, San Diego, CA, USA) was determined by similar method. Apoptosis in tissues was detected using a commercially available TUNEL kit (Apop Tag Peroxidase in situ Apoptosis Detection Kit; Chemicon, Danvers, MA, USA). Detection of PCNA, CD31 and TUNEL was done as described previously $(27,28)$.

Quantification of PCNA, TUNEL and CD31. PCNA and CD31 expression, and TUNEL staining were quantified by counting the numbers of positive cells in 20 randomly chosen fields at x400 (PCNA, TUNEL) or x200 (CD31) magnification. A single microvessel was defined as an individual cell or discrete cluster of CD31-positive cells. The presence of a lumen was not required for being scored as a microvessel.

Statistics. Data are expressed as means \pm standard deviations. Survival of GFP-gCSC-implanted mice (from monoculture and GS-MSLC co-culture groups) was evaluated using the Kaplan-Meier method. Comparisons of cell counts, tumor volume and PCNA, TUNEL and CD31 staining were analyzed by Kruskal-Wallis test. All statistical analyses and graphing were performed using SPSS version 18.0KO software (SPSS Korea, Seoul, Korea).

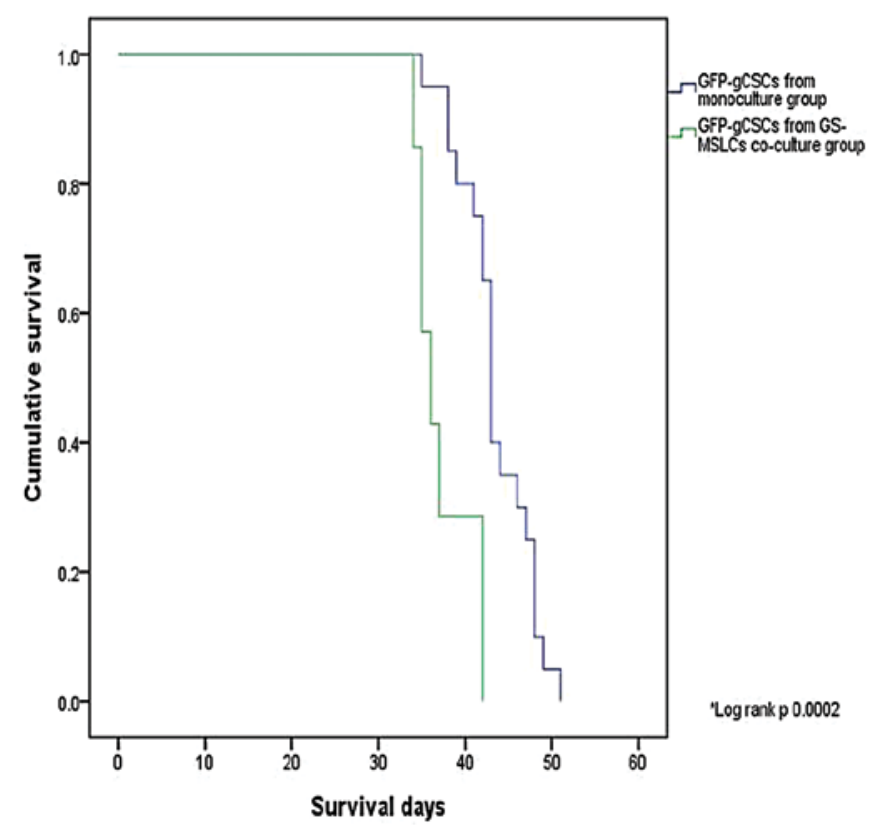

Figure 3. Survival curves of mice injected with GFP-gCSCs isolated from monocultures or GS-MSLC co-cultures. The survival of mice injected with GFP-gCSCs from GS-MSLC co-cultures (37.3 \pm 1.3 days) was significantly shorter compared to that of mice injected with monocultured GFP-gCSCs $(43.5 \pm 1.0$ days; $\mathrm{p}=0.002)$.

\section{Results}

Sorting and counting GFP-gCSCs in vitro. To analyze the effects of GS-MSLCs on the growth of gCSCs in vitro, we cultured GFP-gCSCs alone and together with GS-MSLCs for 3 weeks and counted the numbers of GFP-gCSCs under a fluorescence microscope using a hemocytometer. As shown in Fig. 2, the number of GFP-gCSCs in the group co-cultured with GS-MSLCs $\left(7.76 \pm 0.39 \times 10^{5}\right)$ was significantly increased ( $\sim$-fold) compared to that in the GFP-gCSC monoculture group $\left(2.62 \pm 0.44 \times 10^{5} ; \mathrm{p}=0.002\right)$.

Survival of orthotopic xenograft model mice. To evaluate the effect of GS-MSLCs on in vivo tumor growth, we used an orthotopic xenograft mouse model of glioma. We first compared survival of nude mice cranially implanted with monocultured GFP-gCSCs $(n=20)$ or GFP-gCSCs co-cultured with GS-MSLCs $(n=7)$. As shown in Fig. 3, average survival was significantly decreased in mice injected with GFP-gCSCs co-cultured with GS-MSLCs compared with mice injected with monocultured GFP-gCSCs $(37.3 \pm 1.3$ vs. $43.5 \pm 1.0$ days; $\mathrm{p}=0.002$, log-rank test). No mice implanted with monocultured GFP-gCSCs showed a shorter survival than mice implanted with GFP-gCSCs co-cultured with GS-MSLCs.

Tumor volumes in orthotopic xenograft model mice. Four mice from each GFP-gCSC-implanted group (monoculture and GS-MSLC co-culture) were used to study the effects of GS-MSLCs on the rate and size of gCSC tumor growth in vivo. On day 40 after GFP-gCSCs injection, mouse brains were carefully removed and tumor volumes were measured. 

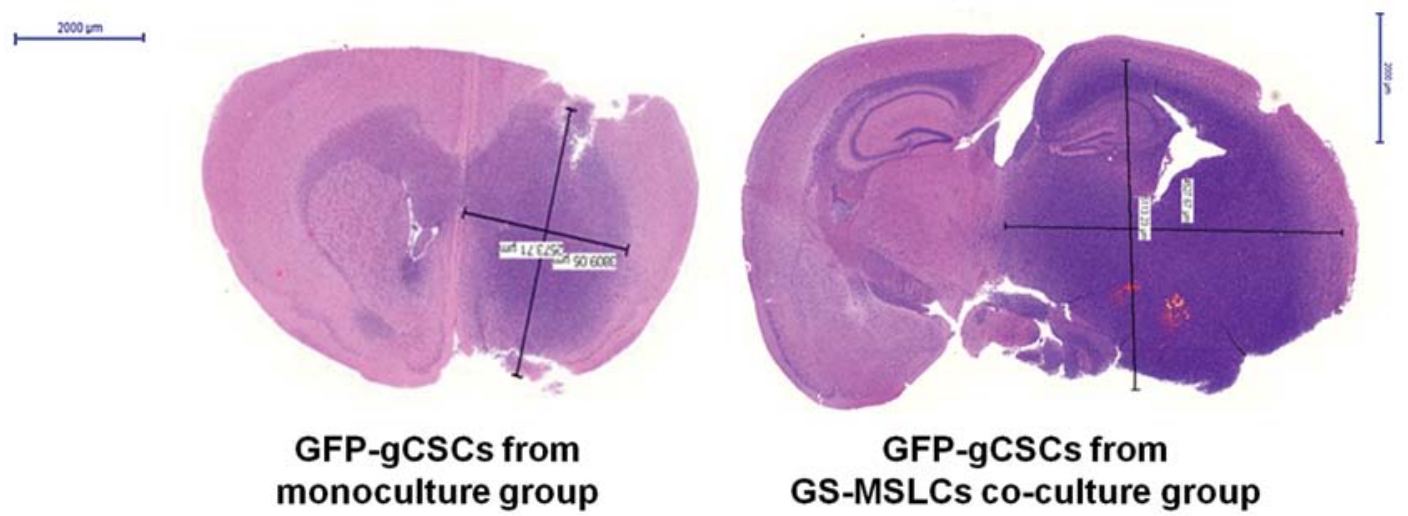

Figure 4. Axial dimensions of the largest tumor portion of mouse brains implanted with GFP-gCSCs obtained from monocultures or GS-MSLC co-cultures. Hematoxylin-stained brain slices show lengths and widths of the largest tumor portion of representative mouse brains implanted with monocultured GFP-gCSCs $(3,809.05 \times 2,573.71 \mu \mathrm{m})$ or GFP-gCSCs isolated from GS-MSLC co-cultures $(4,527.67 \times 5,113.23 \mu \mathrm{m})$.

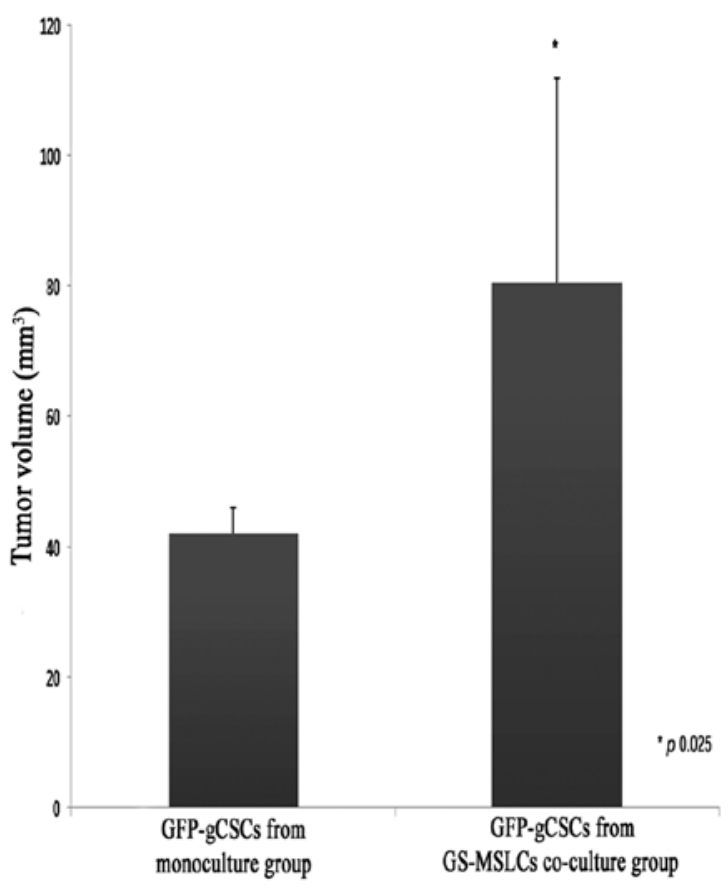

Figure 5. Comparison of the tumor volumes between the brains of mice injected with GFP-gCSCs isolated from monocultures $(n=4)$ or GS-MSLC co-cultures $(\mathrm{n}=4)$. Tumor volumes in mice implanted with GFP-gCSCs from GS-MSLC co-cultures $\left(80.58 \pm 31.29 \mathrm{~mm}^{3}\right)$ were significantly larger than those in mice implanted with GFP-gCSCs cultured alone $\left(42 \pm 4 \mathrm{~mm}^{3} ; \mathrm{p}=0.025\right)$.

Consistent with the survival data, tumors in mice implanted with GFP-gCSCs from GS-MSLC co-cultures were larger in both dimensions (length and width) than those from mice implanted with monocultured GFP-gCSCs (Fig. 4). As shown in Fig. 5, average brain tumor volumes, calculated as described in Materials and methods, were significantly greater in mice implanted with GFP-gCSCs co-cultured with GS-MSLCs $\left(80.58 \pm 31.29 \mathrm{~mm}^{3}\right)$ than in those implanted with GFP-gCSCs cultured alone $\left(42 \pm 4 \mathrm{~mm}^{3} ; \mathrm{p}=0.025\right)$. These data indicate that tumor formation and growth were more rapid in mice injected with GFP-gCSCs co-cultured with GS-MSLCs.
Immunohistochemical analysis of PCNA, TUNEL and CD31. To investigate the biochemical mechanism underlying the in vivo effects of gCSC/GS-MSLC co-culture, we examined tumor cell proliferation (PCNA), apoptosis (TUNEL) and microvessel formation (CD31) using immunohistochemistry (Fig. 6A). The eight mice used for measuring tumor volume were used for these analyses. The number of PCNA-positive cells, an indicator of the rate of proliferation, was not significantly different between tumors formed by GFP-gCSCs cultured alone $(410 \pm 120)$ and GFP-gCSCs co-cultured with GS-MSLC (380 \pm 40 ; Fig. 6B). The number of TUNEL-positive tumor cells, indicative of apoptosis, was also statistically indistinguishable between GFP-gCSCs cultured alone $(151 \pm 36.2)$ and those co-cultured with GS-MSLCs $(222 \pm 84.6$; Fig. 6C). In contrast, the expression of CD31, an indicator of tumor microvessels, was approximately 2.5 -fold higher in tumors formed by GFP-gCSCs from GS-MSLC co-cultures $(350 \pm 50)$ than in those formed from GFP-gCSCs monocultures $(140 \pm 20 ; p=0.016$; Fig. 6D), suggesting increased tumor microvessel density. Collectively, these data imply that co-culture with GS-MSLCs increases the angiogenesis capacity of gCSCs.

\section{Discussion}

In this study, we tested the effects of GS-MSLCs on tumor growth by specifically examining how GS-MSLCs in the tumor microenvironment influence the biological properties of gCSCs. We found that co-culture with GS-MSLCs increased the aggressiveness of gCSCs, as evidenced by their increased cell numbers in vitro, reduced survival of mice orthotopically xenografted, and increased microvessel density in tumors formed from them. Our findings suggest that GS-MSLCs influence the properties of gCSCs, shifting them towards a more aggressive state. Moreover, angiogenesis may be a pivotal part of this mechanism.

Our immunohistochemistry data showed increased CD31 expression in GFP-gCSCs after co-culturing with GS-MSLCs, suggesting increased vessel formation. Since angiogenesis is a hallmark of tumor progression, we believe that, by enhancing the angiogenic capacity of gCSCs, GS-MSLCs might have 
$\mathbf{A}$

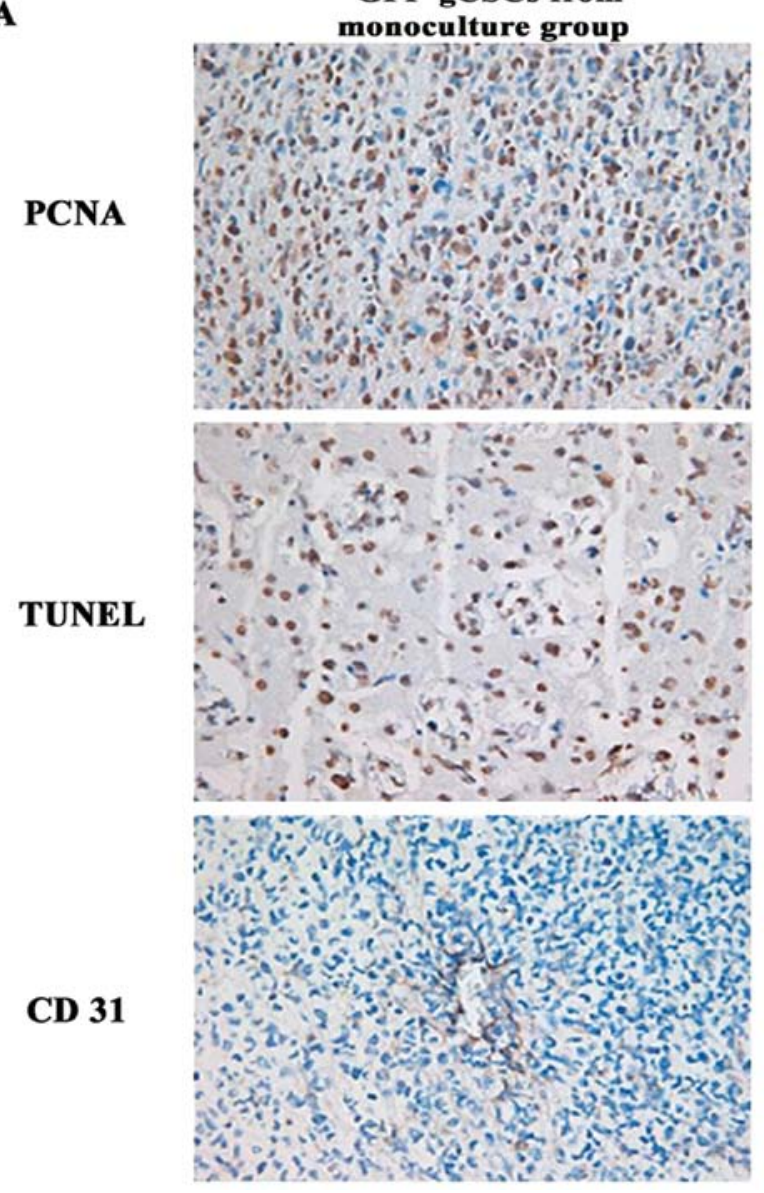

GFP-gCSCs from GS-MSLCSs co-culture group
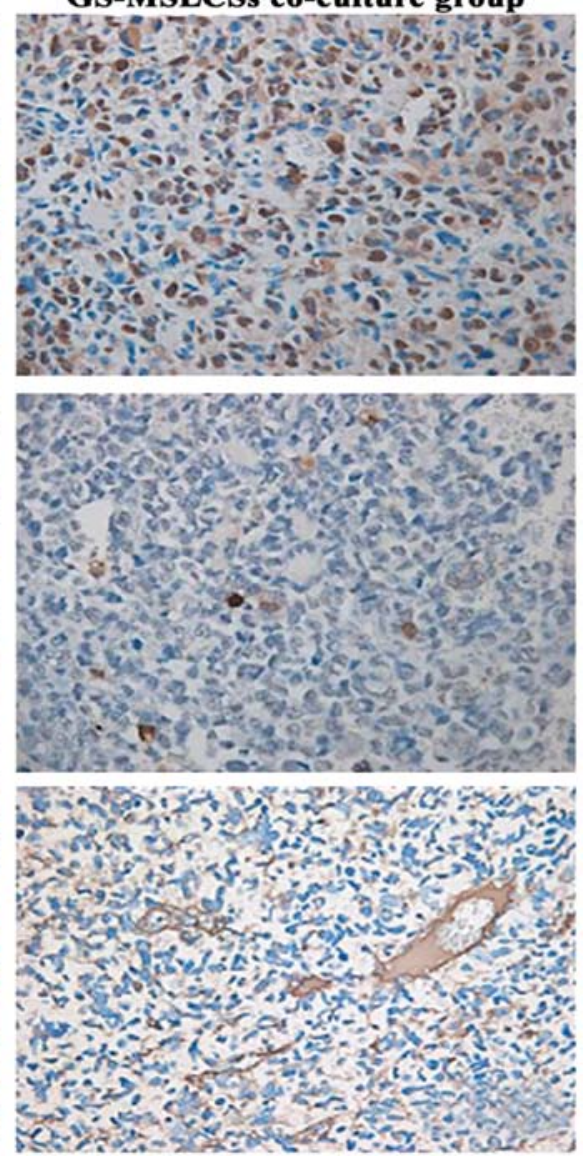

B 600

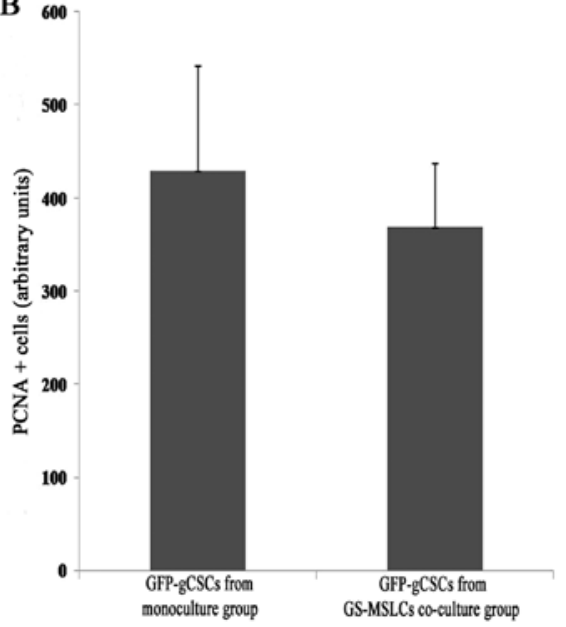

C

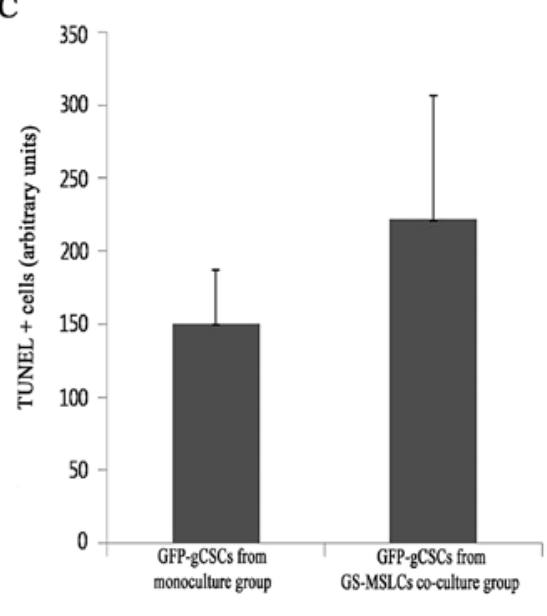

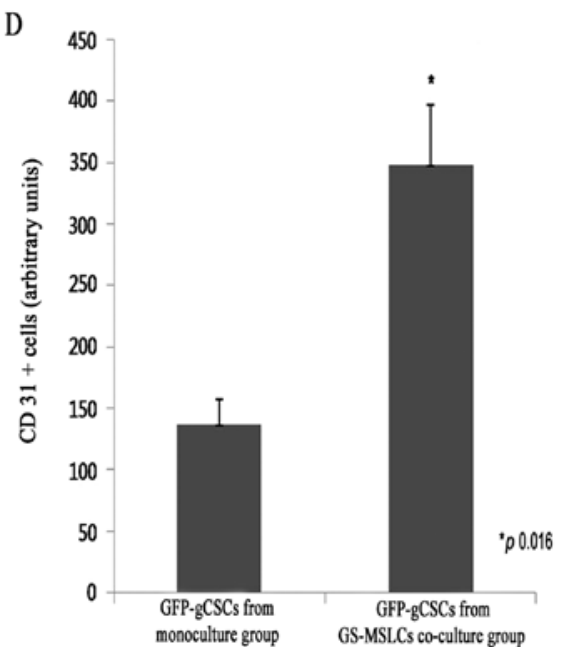

Figure 6. Immunohistochemical analysis of PCNA, TUNEL and CD31. (A) Tumor cell proliferation (PCNA expression), apoptosis (TUNEL staining) and microvessel density (CD31 expression) were assessed immunocytochemically. Original magnification, x400 (PCNA and TUNEL) and x200 (CD31). (B) PCNApositive cells were quantified in 20 random fields at $\mathrm{x} 400$ magnification. The number of PCNA-positive cells in tumors from mice implanted with monocultured GFP-gCSCs $(410 \pm 120, n=4)$ was not significantly different from that in tumors formed by GFP-gCSCs obtained from GS-MSLC co-cultures (380 \pm 40 , $n=4)$. (C) TUNEL-positive cells were quantified in 20 random fields at $x 400$ magnification. The numbers of TUNEL-positive cells in tumors from mice implanted with monocultured GFP-gCSCs $(151 \pm 36.2, \mathrm{n}=4)$ was not significantly different from that in tumors formed by GFP-gCSCs obtained from GS-MSLC co-cultures $(222 \pm 84.6, n=4)$. (D) CD31-expressing cells were quantified in 20 random fields at $x 200$ magnification. The number of CD31-expressing cells was significantly greater in tumors from mice implanted with GFP-gCSCs obtained from GS-MSLC co-cultures $(350 \pm 50, \mathrm{n}=4)$ compared to those in mice implanted with monocultured GFP-gCSCs $(140 \pm 2, n=4 ; \mathrm{p}=0.016)$.

contributed to the more rapid and extensive tumor growth in mice implanted with GFP-gCSCs co-cultured with GS-MSLCs (Figs. 5 and 6). The increased number of GFP-gCSCs in co-cultures of gCSCs and GS-MSLCs in vitro (Fig. 2) suggested the possibility that a mechanism involving increased cell proliferation and/or decreased cell death also contributed 
to the more aggressive tumor phenotype. Although the level of proliferation (PCNA expression) showed a higher trend in tumors formed by GFP-gCSCs obtained from GS-MSLC co-cultures, and apoptosis (TUNEL staining) lower trends that would be consistent with the in vitro data-these differences did not reach statistical significance (Fig. 6B and C). Thus, angiogenesis appears to play a critical role in creating a supportive environment for gCSCs and tumor growth (Fig. 6D), other mechanisms may also contribute. Whether a different experimental design and larger sample size might establish a significant role for proliferation and/or apoptosis, or whether some entirely different mechanism is involved, are questions currently under investigation in our laboratory.

Our data strongly argue that GS-MSLCs favorably influence the growth of glioma; however, not all literature reports are in agreement on this point. Qiao et al reported that human MSCs inhibit the proliferation and colony-forming ability of human cancer cell lines (29); a similar antitumoral role of MSCs was also observed in Kaposi's sarcomas (30) and colon carcinoma in rats (31). Furthermore, contrary to our data, Nakamura et al reported that rat MSCs inhibit the growth of 9L glioma cells both in vitro and in vivo and increase the survival of 9L glioma-bearing rats (32). An important point that sets our experiments apart from these data is that we used GS-MSLCs isolated from that GBM stroma, not normal MSCs from a normal organ. We think that it is possible that MSCs in a tumor may be re-programmed or otherwise altered by the tumor microenvironment, changing their characteristics to those that favor tumor growth.

While some researchers have argued that MSCs negatively impact tumor growth, numerous researchers have reported experiments supporting the tumor growth-enhancing role of MSCs. It has been speculated that, by secreting cytokines that mediate angiogenesis and immunosuppressive effects in tumor sites, MSCs provide a stem cell niche that facilitates tumor progression (33-36). In a paper on the suppressive role of MSCs in hematopoietic and non-hematopoietic tumor cells in vitro, Ramasamy et al also demonstrated that co-injection of MSCs with tumor cells resulted in more rapid in vivo tumor growth (37). Tumor cell-protecting and growth-promoting roles of MSCs have also been observed in many other cancers, including melanoma $(15,38)$, breast cancer (39), myeloid leukemia (40) and colon cancer (41). Furthermore, MSCs have also been shown to stimulate metastasis of breast cancer (6) and play a role in the drug resistance of leukemia cells (42-44).

Despite our data and other evidence suggesting tumor progression-enhancing role for the MSCs and MSLCs, negative results on this phenomenon are not readily dismissed. The reasons for these discrepancies are not fully understood, but Sasser et al (45) and Karnoub et al (6) have proposed that the genetic background of tumor cells may be a key determinant in the MSCs-tumor relationship. Specifically, Sasser et al reported that an MSC paracrine factor enhanced the growth of estrogen receptor- $\alpha(E R \alpha)$-positive breast cancer, but had no or a substantially diminished effect on ER $\alpha$-negative breast cancer (45). Similarly, Karnoub et al found that MSCs only accelerated the growth of ER $\alpha$-positive, but not ER $\alpha$-negative, tumor xenografts in mouse skin (6). Although the mechanism underlying this apparent duality of MSL/MSLC effects remains to be elucidated, it is clear that the MSCs or MSLCs microenvironment is a double-edged sword capable of exacerbating cancer by stimulating its progression, metastasis, and resistance to drugs (46).

Mesenchymal fibroblasts within solid tumors, referred to as activated fibroblasts or carcinoma-associated fibroblasts (CAFs), serve as indirect evidence supporting tumor-promoting role of MSCs or MSLCs. Although the origin and biology of CAFs are not completely defined, the fact that CAFs and MSCs share common surface markers and exhibit similar functions implies that they may originate from the same progenitor cell $(47,48)$ or that MSCs may differentiate into CAFs in a tumor context (41,49-51). The hypothesis that MSCs transition into CAFs within a tumor environment is supported by a study by Shinagawa et al (41). These authors observed MSCs moving towards the stroma of colon cancer tumors and differentiating into CAFs, and thereby promoting angiogenesis, tumor growth, migration, invasion, and metastasis. Considerable additional experimental evidence has definitively shown that CAFs play a role in the progression towards an aggressive phenotype in various cancers (52-56). Although these results do not unambiguously establish that MSCs differentiate into CAFs, they strongly suggest that MSCs and CAFs are closely associated and indicate that CAFs might participate in the mechanism by which MSCs modulate the tumor microenvironment. The possibility that GS-MSLCs may also interact with CAFs in glioma microenvironment suggested by these reports is currently under investigation in our laboratory.

In our experiments, gCSCs and GS-MSLCs were in direct contact when co-cultured, allowing bidirectional crosstalk between them. Karnoub et al have also pointed out that the increase in the metastatic potency of tumor cells induced by MSCs requires that the two cell types be in direct contact (6). This phenomenon is consistent with the current understanding that MSCs interact with tumor cells by direct contact and though paracrine factors. Tumor cells secrete cytokines that attract MSCs to the tumor stroma and MSCs release cytokines, matrix-degrading enzymes, and immunomodulatory factors that regulate tumor growth, invasion and metastasis $(5,6,57)$. We believe that the direct contact between MSCs or MSLCs and gCSCs in our experimentational setting mimics that of the in vivo tumor microenvironment. In fact gCSCs $(22,58)$ and GS-MSLCs $(12,13)$ both reside within vascular niches in gliomas. Although we are not yet certain whether GS-MSLCs and normal MSCs share the same functions and interact with gCSCs in the same manner, the fact that they reside in the same site strengthens the possibility for cross-talk between gCSCs and GS-MSLCs through direct contact and paracrine factors.

The tumor-homing characteristics of MSCs have led many researchers to investigate the potential of MSCs as targeted delivery vehicles for cancer therapy $(59,60)$. The results of our experiments, however, offer a cautionary tale. Although normal MSCs and GS-MSLCs are not identical, our demonstration that co-culture with GS-MSLCs increases gCSC cell numbers in vitro, reduces the survival of mice injected with gCSCs co-cultured with GS-MSLCs, and increases tumor volume and vessel formation in the tumors of these mice warns of the potential dangers of MSC-gCSC interactions. These concerns notwithstanding, MSCs still have great potential as a therapeutic tool in cancer, but more likely as a therapeutic target rather than as a targeted-delivery vehicle. MSCs are known to affects the biological characteristics of cancer by 
three mechanisms: i) production of growth factors, cytokines, and immunomodulatory factors that favor tumor progression; ii) formation of a cancer stem cell niche that facilitates the growth of cancer stem cells; and iii) differentiation into other stromal cells, such as CAFs. Our data further suggest that GS-MSLCs may even shift the biological property of cancer stem cells toward a more malignant phenotype. Although it is clear that considerable additional study will be required before this mechanism is completely understood and can be applied clinically, GS-MSLCs and their interactions with tumor cells are a promising new target in cancer therapy.

In conclusion, we found that GS-MSLCs, as a component of the tumor microenvironment, significantly change the biological properties of gCSCs. Co-culture with GS-MSLCs increased the aggressiveness of gCSCs, as evidenced by elevated cell counts in vitro, shortened survival time in orthotopic xenograft mouse models, and increased tumor volume and vessel formation in vivo. Moreover, from the increased expression of the endothelial cell marker CD31, we infer that GS-MSLCs positively influence tumor growth by enhancing the angiogenic capacity of gCSCs. The next step is to clarify the exact mechanism underlying the supportive relationship between GS-MSLCs and gCSCs. These experiments are currently underway in our laboratory.

\section{Acknowledgements}

This research was supported by the Basic Science Research Program through the National Research Foundation of Korea (NRF) funded by the Ministry of Education, Science and Technology (2010-0004506) and a grant from the National R\&D Program for Cancer Control, Ministry for Health, Welfare and Family Affairs, Republic of Korea (1020340).

\section{References}

1. Fidler IJ and Poste G: The 'seed and soil' hypothesis revisited. Lancet Oncol 9: 808, 2008.

2. Poste G and Fidler IJ: The pathogenesis of cancer metastasis. Nature 283: 139-146, 1980.

3. Paget S: The distribution of secondary growths in cancer of the breast. Lancet 133: 571-573, 1889.

4. Albini A and Sporn MB: The tumour microenvironment as a target for chemoprevention. Nat Rev Cancer 7: 139-147, 2007.

5. Zhang W, Remenyik E, Zelterman D, Brash DE and Wikonkal NM: Escaping the stem cell compartment: sustained UVB exposure allows p53-mutant keratinocytes to colonize adjacent epidermal proliferating units without incurring additional mutations. Proc Natl Acad Sci USA 98: 13948-13953, 2001.

6. Karnoub AE, Dash AB, Vo AP, et al: Mesenchymal stem cells within tumour stroma promote breast cancer metastasis. Nature 449: 557-563, 2007.

7. El-Haibi CP and Karnoub AE: Mesenchymal stem cells in the pathogenesis and therapy of breast cancer. J Mammary Gland Biol Neoplasia 15: 399-409, 2011.

8. Lin TM, Chang HW, Wang KH, et al: Isolation and identification of mesenchymal stem cells from human lipoma tissue. Biochem Biophys Res Commun 361: 883-889, 2007.

9. Houghton J, Stoicov C, Nomura S, et al: Gastric cancer originating from bone marrow-derived cells. Science 306 1568-1571, 2004.

10. Gibbs CP, Kukekov VG, Reith JD, et al: Stem-like cells in bone sarcomas: implications for tumorigenesis. Neoplasia 7 : 967-976, 2005

11. Kang SG, Shinojima N, Hossain A, et al: Isolation and perivascular localization of mesenchymal stem cells from mouse brain. Neurosurgery 67: 711-720, 2010
12. Kim SM, Kang SG, Park NR, et al: Presence of glioma stroma mesenchymal stem cells in a murine orthotopic glioma model. Childs Nerv Syst 27: 911-922, 2011.

13. Kim YG, Jeon S, Sin GY, et al: Existence of glioma stroma mesenchymal stem-like cells in Korean glioma specimens. Childs Nerv Syst: Dec 29, 2012 (Epub ahead of print). doi:10.1007/s00381-00012-01988-00381.

14. Dominici M, Le Blanc K, Mueller I, et al: Minimal criteria for defining multipotent mesenchymal stromal cells. The International Society for Cellular Therapy position statement. Cytotherapy 8: 315-317, 2006.

15. Kucerova L, Matuskova M, Hlubinova $\mathrm{K}$, Altanerova $\mathrm{V}$ and Altaner C: Tumor cell behaviour modulation by mesenchymal stromal cells. Mol Cancer 9: 129, 2010.

16. Shin GY, Shim JK, Lee JH, et al: Changes in the biological characteristics of glioma cancer stem cells after serial in vivo subtransplantation. Childs Nerv Syst 29: 55-64, 2013.

17. Dirks PB: Brain tumor stem cells: bringing order to the chaos of brain cancer. J Clin Oncol 26: 2916-2924, 2008.

18. Galli R, Binda E, Orfanelli U, et al: Isolation and characterization of tumorigenic, stem-like neural precursors from human glioblastoma. Cancer Res 64: 7011-7021, 2004.

19. Singh SK, Hawkins C, Clarke ID, et al: Identification of human brain tumour initiating cells. Nature 432: 396-401, 2004.

20. Sulman E, Aldape $K$ and Colman H: Brain tumor stem cells. Curr Probl Cancer 32: 124-142, 2008.

21. Yuan X, Curtin J, Xiong Y, et al: Isolation of cancer stem cells from adult glioblastoma multiforme. Oncogene 23: 9392-9400, 2004.

22. Kong BH, Park NR, Shim JK, et al: Isolation of glioma cancer stem cells in relation to histological grades in glioma specimens. Childs Nerv Syst 29: 217-219, 2013.

23. Louis DN, Ohgaki H, Wiestler OD, et al: The 2007 WHO classification of tumours of the central nervous system. Acta Neuropathol 114: 97-109, 2007.

24. Singh SK, Clarke ID, Terasaki M, et al: Identification of a cancer stem cell in human brain tumors. Cancer Res 63: 5821-5828, 2003.

25. Lal S, Lacroix M, Tofilon P, Fuller GN, Sawaya R and Lang FF: An implantable guide-screw system for brain tumor studies in small animals. J Neurosurg 92: 326-333, 2000.

26. Nam DH, Park K, Park C, et al: Intracranial inhibition of glioma cell growth by cyclooxygenase-2 inhibitor celecoxib. Oncol Rep 11: 263-268, 2004.

27. Kang SG, Kim JS, Park K, Kim JS, Groves MD and Nam DH: Combination of celecoxib and temozolomide in C6 rat glioma orthotopic model. Oncol Rep 15: 7-13, 2006.

28. Kim SJ, Uehara H, Karashima T, Shepherd DL, Killion JJ and Fidler IJ: Blockade of epidermal growth factor receptor signaling in tumor cells and tumor-associated endothelial cells for therapy of androgen-independent human prostate cancer growing in the bone of nude mice. Clin Cancer Res 9: 1200-1210, 2003.

29. Qiao L, Xu Z, Zhao T, et al: Suppression of tumorigenesis by human mesenchymal stem cells in a hepatoma model. Cell Res 18: 500-507, 2008

30. Khakoo AY, Pati S, Anderson SA, et al: Human mesenchymal stem cells exert potent antitumorigenic effects in a model of Kaposi's sarcoma. J Exp Med 203: 1235-1247, 2006.

31. Ohlsson LB, Varas L, Kjellman C, Edvardsen K and Lindvall M: Mesenchymal progenitor cell-mediated inhibition of tumor growth in vivo and in vitro in gelatin matrix. Exp Mol Pathol 75: 248-255, 2003.

32. Nakamura K, Ito Y, Kawano Y, et al: Antitumor effect of genetically engineered mesenchymal stem cells in a rat glioma model. Gene Ther 11: 1155-1164, 2004.

33. Roorda BD, ter Elst A, Kamps WA and de Bont ES: Bone marrow-derived cells and tumor growth: contribution of bone marrow-derived cells to tumor micro-environments with special focus on mesenchymal stem cells. Crit Rev Oncol Hematol 69: 187-198, 2009.

34. Djouad F, Plence P, Bony C, et al: Immunosuppressive effect of mesenchymal stem cells favors tumor growth in allogeneic animals. Blood 102: 3837-3844, 2003.

35. Le Blanc K and Ringden O: Immunobiology of human mesenchymal stem cells and future use in hematopoietic stem cell transplantation. Biol Blood Marrow Transplant 11: 321-334, 2005.

36. Zhu W, Xu W, Jiang R, et al: Mesenchymal stem cells derived from bone marrow favor tumor cell growth in vivo. Exp Mol Pathol 80: 267-274, 2006. 
37. Ramasamy R, Lam EW, Soeiro I, Tisato V, Bonnet D and Dazzi F: Mesenchymal stem cells inhibit proliferation and apoptosis of tumor cells: impact on in vivo tumor growth. Leukemia 21: 304-310, 2007.

38. Wang $X$, Zhang $Z$ and Yao C: Survivin is upregulated in myeloma cell lines cocultured with mesenchymal stem cells. Leuk Res 34: 1325-1329, 2010.

39. Patel SA, Meyer JR, Greco SJ, Corcoran KE, Bryan M and Rameshwar P: Mesenchymal stem cells protect breast cancer cells through regulatory $\mathrm{T}$ cells: role of mesenchymal stem cell-derived TGF-beta. J Immunol 184: 5885-5894, 2010.

40. Konopleva M, Konoplev S, Hu W, Zaritskey AY, Afanasiev BV and Andreeff M: Stromal cells prevent apoptosis of AML cells by up-regulation of anti-apoptotic proteins. Leukemia 16 : 1713-1724, 2002

41. Shinagawa K, Kitadai Y, Tanaka M, et al: Mesenchymal stem cells enhance growth and metastasis of colon cancer. Int J Cancer 127: 2323-2333, 2010.

42. Lin YM, Zhang GZ, Leng ZX, et al: Study on the bone marrow mesenchymal stem cells induced drug resistance in the U937 cells and its mechanism. Chin Med J (Engl) 119: 905-910, 2006.

43. Kurtova AV, Balakrishnan K, Chen R, et al: Diverse marrow stromal cells protect CLL cells from spontaneous and druginduced apoptosis: development of a reliable and reproducible system to assess stromal cell adhesion-mediated drug resistance. Blood 114: 4441-4450, 2009.

44. Vianello F, Villanova F, Tisato V, et al: Bone marrow mesenchymal stromal cells non-selectively protect chronic myeloid leukemia cells from imatinib-induced apoptosis via the CXCR4/CXCL12 axis. Haematologica 95: 1081-1089, 2010.

45. Sasser AK, Mundy BL, Smith KM, et al: Human bone marrow stromal cells enhance breast cancer cell growth rates in a cell line-dependent manner when evaluated in 3D tumor environments. Cancer Lett 254: 255-264, 2007.

46. Zhang W: Mesenchymal stem cells in cancer: friends or foes. Cancer Biol Ther 7: 252-254, 2008.

47. Haniffa MA, Wang XN, Holtick U, et al: Adult human fibroblasts are potent immunoregulatory cells and functionally equivalent to mesenchymal stem cells. J Immunol 179: 15951604, 2007.

48. Paunescu V BF, Tatu CA, Gavriliuc OI, Rosca A, Gruia AT, Tanasie G, Bunu C, Crisnic D, Gherghiceanu M, Tatu FR, Tatu CS and Vermesan S: Tumour-associated fibroblasts and mesenchymal stem cells: more similarities than differences. J Cell Mol Med 15: 635-646, 2011.
49. Spaeth EL, Dembinski JL, Sasser AK, et al: Mesenchymal stem cell transition to tumor-associated fibroblasts contributes to fibrovascular network expansion and tumor progression. PLoS One 4: e4992, 2009.

50. Mishra PJ, Humeniuk R, Medina DJ, et al: Carcinoma-associated fibroblast-like differentiation of human mesenchymal stem cells. Cancer Res 68: 4331-4339, 2008.

51. Hall B, Dembinski J, Sasser AK, Studeny M, Andreeff M and Marini F: Mesenchymal stem cells in cancer: tumor-associated fibroblasts and cell-based delivery vehicles. Int J Hematol 86: 8-16, 2007.

52. Brentnall TA, Lai LA, Coleman J, Bronner MP, Pan S and Chen R: Arousal of cancer-associated stroma: overexpression of palladin activates fibroblasts to promote tumor invasion. PLoS One 7: e30219, 2012.

53. Zhi K, Shen X, Zhang H and Bi J: Cancer-associated fibroblasts are positively correlated with metastatic potential of human gastric cancers. J Exp Clin Cancer Res 29: 66, 2010.

54. Liao D, Luo Y, Markowitz D, Xiang R and Reisfeld RA: Cancer associated fibroblasts promote tumor growth and metastasis by modulating the tumor immune microenvironment in a 4T1 murine breast cancer model. PLoS One 4: e7965, 2009.

55. Casey TM, Eneman J, Crocker A, et al: Cancer associated fibroblasts stimulated by transforming growth factor beta1 (TGF-beta 1) increase invasion rate of tumor cells: a population study. Breast Cancer Res Treat 110: 39-49, 2008.

56. Ostman A and Augsten M: Cancer-associated fibroblasts and tumor growth - bystanders turning into key players. Curr Opin Genet Dev 19: 67-73, 2009.

57. Honczarenko M, Le Y, Swierkowski M, Ghiran I, Glodek AM and Silberstein LE: Human bone marrow stromal cells express a distinct set of biologically functional chemokine receptors. Stem Cells 24: 1030-1041, 2006.

58. Gilbertson RJ and Rich JN: Making a tumour's bed: glioblastoma stem cells and the vascular niche. Nat Rev Cancer 7: 733-736, 2007.

59. Ciavarella S, Dominici M, Dammacco F and Silvestris F: Mesenchymal stem cells: a new promise in anticancer therapy. Stem Cells Dev 20: 1-10, 2011.

60. Dai LJ, Moniri MR, Zeng ZR, Zhou JX, Rayat J and Warnock GL: Potential implications of mesenchymal stem cells in cancer therapy. Cancer Lett 305: 8-20, 2011. 